\title{
Artículos
}

\section{Las comunidades virtuales académicas y científicas españolas: el caso de Redlris}

\author{
Por José Antonio Ontalba y Ruipérez
}

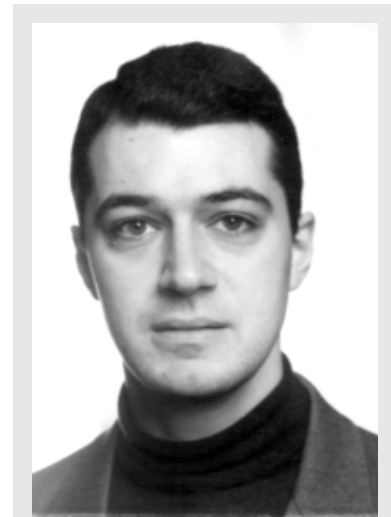

José A ntonio O ntalba y Ruipérez

Resumen: Las comunidades virtuales de usuarios (o CVU) han devenido una plataforma óptima para el trabajo cooperativo y la investigación colaborativa. El presente trabajo describe el desarrollo de las CVUs académicas y científicas españolas de RedIris a partir de su oferta de herramientas y servicios de información. Los resultados muestran una gran dependencia de las listas de distribución y, en general, una infrautilización de las CVUs y de sus servicios.

Descriptores: Comunicación científica, Servicios de información, Comunidades virtuales, Comunidades en línea, CVU, Trabajo colaborativo, Groupware.

Title: Spanish virtual academic and scientific communities. The case of Redlris.

Abstract: Virtual communities of users (VCU) have become an ideal platform for co-operative work and collaborative research. This article describes developments towards Spanish academic and scientific VCUs undertaken by RedIris [the Spanish National Research Network] through the information tools and services it provides. The results show a great dependence on distribution lists and, in general, a low use of VCUs and their services.

Keywords: Scientific communication, Information services, Virtual communities, Online communities, VCU, Collaborative work, GroupWare.

Ontalba y Ruipérez, José Antonio. "Las comunidades virtuales académicas y científicas españolas: el caso de RedIris". En: El profesional de la información, 2002, septiembre-octubre, v. 11, n. 5, pp. 328-338.

\section{Introduc ción}

Las comunidades virtuales de usuarios (o CVUs) han sido, hasta la fecha, objeto de análisis desde perspectivas ajenas al área de la biblioteconomía y la documentación. Así, por ejemplo, la sociología ha analizado (desde su relación con la psicología) el problema de la identidad en línea y cómo la tecnología ha incidido en nuevas formas de relación social. La informática ha visto en las comunidades virtuales un área de integración de desarrollos que van desde la comunicación mediada por ordenador hasta la usabilidad, pasando por la gestión de bases de datos, por ejemplo. Por otro lado, desde las ciencias de la educación se han detectado nuevas posibilidades y oportunidades que les ofrece el proceso de enseñanza-aprendizaje en este entorno digital. Finalmente, desde el ámbito de la empresa, se han valorado las comunidades virtuales como elementos que fomentan y potencian el capital intelectual por medio del intercambio de conocimientos y ex- periencias personales o corporativas entre miembros de la entidad.

\section{«El estudio de la CVU desde el punto de vista de la gestión de la información ha sido escaso y muy reciente»}

El estudio de la CVU desde el punto de vista de la gestión de la información ha sido escaso y, en España, recentísimo ${ }^{1}$. En cualquier caso, las más de las veces se ha hecho de la mano de la gestión del conocimiento, aprovechando que es un fenómeno en boga y que, al implicar a las empresas, suele llevar parejo cierta inversión de capital. Sin embargo, el enfoque desde, para, por y hacia el mundo académico y científico se puede decir que ha sido nulo. Tanto más en lo que se refiere a España o al ámbito hispanohablante.

Este artículo, pues, tiene como objetivo estudiar y analizar las herramientas de información y comunica- 


\begin{tabular}{|c|c|c|c|c|c|c|c|c|c|c|c|c|c|c|c|}
\hline & Ort. & Der. & Tier. & Neur. & T-Ed & CGA & Astr. & P-Ed & Farm & Dep. & Ent. & ocs & AMMT & $\mathrm{PC}$ & cs \\
\hline \multicolumn{16}{|c|}{ HERRAMIENTAS DE INFORMACIÓN } \\
\hline \multicolumn{16}{|l|}{ INTERNA } \\
\hline Inf sobre la cvu & * & * & & * & * & * & * & & * & & * & * & * & * & * \\
\hline Directorio de miembros & & * & & * & & & & & & & * & & & & \\
\hline Estadísticas de acceso & * & * & $*$ & & * & & * & & & & & * & & * & * \\
\hline Novedades & & & & & & & & & & & * & * & * & & \\
\hline Mapa de la sede & & & & & & & & & & & $*$ & & & & \\
\hline Buscador interno & & & $*$ & & $*$ & * & & & & & $*$ & & $*$ & * & * \\
\hline \multicolumn{16}{|l|}{ EXTERNA } \\
\hline Novedades externas & * & * & * & * & * & & & & * & & * & & * & & * \\
\hline Documentación a texto completo & * & * & * & * & * & & & * & * & & * & * & & $*$ & \\
\hline Bases de datos & * & & & & & & & & & & & & & * & \\
\hline Bibliografia & & & * & & * & & & & & & & * & & $*$ & \\
\hline Recopilación de enlaces & $*$ & $*$ & $*$ & $*$ & $*$ & $*$ & $*$ & * & $*$ & * & $*$ & $*$ & $*$ & $*$ & \\
\hline \multicolumn{16}{|c|}{ HERRAMIENTAS DE COMUNICACIÓN } \\
\hline Listas de correo & * & * & $*$ & * & $*$ & $*$ & & * & * & * & * & & & * & $*$ \\
\hline Foro de debate & & $*$ & $*$ & $*$ & & & & & & & $*$ & $*$ & & & \\
\hline Eventos virtuales & & & & $*$ & & * & & & & & & $*$ & & & \\
\hline Chat & & & $*$ & * & & & & & & & $*$ & $*$ & & & \\
\hline Zona de trabajo & & & & & $*$ & $*$ & & & $*$ & & $*$ & & $*$ & & $*$ \\
\hline \multicolumn{16}{|l|}{ SERVICIOS } \\
\hline Cursos virtuales & & & & $*$ & & & & & & & $*$ & & $*$ & $*$ & $*$ \\
\hline Alojamiento de página web & & & $*$ & & & & & & & & * & $*$ & & $*$ & \\
\hline Revista electrónica & & $*$ & & & $*$ & & & & & * & $*$ & $*$ & & $*$ & $*$ \\
\hline
\end{tabular}

Acrónimos utilizados:

Ort.: Ortopedi@. Cirugia Ortopédica y Traumatologia

Der.: Derecons. Red Académica de Derecho Constitucional

Tier.: Tierra. Red Temática de Ciencias de la Tierra de España

Neur.: Comunidad Virtual de Neurologia

T-Ed Tecnologias Educativas

CGA: Comunidad Virtual de las Ciencias de la Gestión del Agua

Astr:: Astrobionet. Comunidad Virtual de Astrobiologia
P-Ed Psicología en Educación

Farm: Farmatoxi. Comunidad Virtual de Farmacología y Toxicologia

Dep.: Comunidad Virtual de Ciencias del Deporte

Ent.: Comunidad Virtual de Entomologia

OCS : Observatorio para la CiberSociedad

AMMT. Área de Máquinas y Motores Térmicos

PC: Proyecto Clio

CS : Cálculo Simbólico

Tabla 1: Presencia de herramientas y servicios de información y comunicación en las CVUs seleccionadas

ción empleadas por las CVUs del área científica-académica en España y, más concretamente, de una muestra de las CVUs que integran la red académica española RedIris ${ }^{2}$.

Hasta hace pocos años, RedIris ofrecía la lista de distribución como única herramienta para el trabajo cooperativo y en grupo al colectivo de usuarios de la Red con un mismo perfil académico o científico y unos objetivos e intereses comunes.

Ante las más que notables limitaciones de las listas en aquellos casos en que han quedado inoperantes desbordadas por el número de miembros y la falta de una masa crítica mínima que aporte cierto contenido ${ }^{3}$, RedIris ofrece sus servicios de comunidades virtuales de usuarios con, principalmente, los siguientes objetivos ${ }^{4}$ :

- Ofrecer a todos los usuarios de la comunidad RedIris interesadas en temáticas concretas un punto de encuentro, información y coordinación, en definitiva, un servicio integrado por encima de barreras geográficas que satisfaga las necesidades telemáticas y de coordinación de estos grupos de interés.

- Colaborar técnicamente a la unión y coordinación entre científicos involucrados en una misma temática para elaborar estrategias y proyectos de investigación.
- Servir de nexo de unión y colaboración con las asociaciones o sociedades científicas españolas vinculadas al colectivo científico español, así como mejorar la coordinación con los diferentes Programas Nacionales de Investigación y Desarrollo.

-Posibilidad de mecenazgo de actividades o experimentos dentro de una CVU.

En resumidas cuentas, con todo ello se busca satisfacer la necesidad de aumentar el nivel cuantitativo y cualitativo de las aplicaciones ofertadas a los grupos articulados alrededor de las listas de distribución de RedIris, permitiéndoles a éstas un desarrollo mayor en las CVUs.

\section{Análisis de las CVUs alojadas en RedIris}

\subsection{Material y métodos}

Se presenta un análisis de contenido transversal descriptivo de quince CVUs integradas en RedIris.

De las 34 CVUs que actualmente existen en RedI$r i s^{5}$ se han escogido aquellas que cumplen unos requisitos mínimos en cuanto a estructura, contenidos, naturaleza y funcionamiento actual. Se han descartado aquellas que no eran CVUs, sino revistas electrónicas o páginas de entidades académicas o profesionales; tampoco se han considerado aquellas que no se han ac- 
tualizado en mucho tiempo, las que tenían gran parte de su estructura vacía o no disponible, y las que apenas disponían de accesos (a partir de la consulta de las estadísticas), lo que es sintomático de la vida de éstas.

Conviene advertir que el servicio de CVUs se ofrece organizado en una serie de Áreas temáticas ${ }^{6}$ donde se clasifican los diferentes grupos de interés que han devenido CVU o Red temática ${ }^{7}$, casi todos ellos, como se ha comentado anteriormente, por el desarrollo de una lista distribución de temática pareja. Las limitaciones de éstas han provocado la búsqueda de un servicio con mayores prestaciones por parte de la minoría activa, lo que vendría representado por la comunidad virtual.

\section{«Ante las limitaciones de las listas de distribución cuando quedan inoperantes desborda- das por el número de miem- bros y la falta de una masa crí- tica mínima que aporte cierto contenido, Redlris ofrece sus servicios de CVUs»}

Cabe la posibilidad de que una Red temática forme parte de varias Áreas; así, por ejemplo, Ingeniería romana se encuentra ubicada dentro de la áreas de historia y de ciencias tecnológicas, o Ciencias de gestión del agua se halla en ciencias de la tierra y el espacio, ciencias económicas, ciencias jurídicas y derecho y ciencias políticas.

http://traianus.rediris.es/

http://agua.rediris.es/

En función de todo lo anterior, las CVUs seleccionadas para este estudio son:

—Ortopedi@. Cirugía ortopédica y traumatología:

http://ortopedia.rediris.es/

-Derecons. Red académica de derecho constitucional:

http://constitucion.rediris.es/Princip.html

-Tierra. Red académica de ciencias de la Tierra en España:

http://tierra.rediris.es/

-Comunidad virtual de neurología:

http://neurologia.rediris.es/neurologia/index.html

-Edutec. Tecnologías educativas:

http://edutec.rediris.es/

\begin{tabular}{|l|c|c|}
\hline \multicolumn{1}{|c|}{ HERRAMIENTAS DE INFORMACIÓN } & N $^{\mathbf{0}}$ CVUs & Porcentaje \\
\hline \multicolumn{1}{|c|}{ INTERNA } & $\mathbf{1 5}$ & $\mathbf{1 0 0}$ \\
\hline Información sobre la CVU & 13 & 87 \\
\hline Directorio de miembros & 12 & 80 \\
\hline Estadísticas de acceso & 3 & 20 \\
\hline Novedades & 8 & 53 \\
\hline Mapa de la sede & 3 & 20 \\
\hline Buscador interno EXTERNA & 1 & 7 \\
\hline & 7 & 47 \\
\hline Novedades externas & 15 & 100 \\
\hline Documentación a texto completo & 9 & 60 \\
\hline Bases de datos & 10 & 67 \\
\hline Bibliografia & 2 & 13 \\
\hline Recopilación de enlaces & 5 & 33 \\
\hline \multicolumn{1}{|c|}{ HERRAMIENTAS DE COMUNICACIÓN } & 14 & 93 \\
\hline Listas de correo & $\mathbf{1 4}$ & $\mathbf{9 3}$ \\
\hline Foros de debate & 12 & 80 \\
\hline Eventos virtuales & 5 & 33 \\
\hline Chat & 3 & 20 \\
\hline Zona de trabajo & 4 & 27 \\
\hline \multicolumn{1}{|c|}{ SERVICIOS } & 6 & 40 \\
\hline Cursos virtuales & $\mathbf{1 0}$ & $\mathbf{6 7}$ \\
\hline Alojamiento de página web & 5 & 33 \\
\hline Revista electrónica & 7 & 7 \\
\hline
\end{tabular}

Tabla 2: Uso de las diferentes herramientas y servicios en el conjunto de las CVUs seleccionadas (número de comunidades y porcentaje respecto del total, 15)

- Comunidad virtual de ciencias de la gestión del agua:

http://agua.rediris.es/

-Astrobionet. Comunidad virtual de astrobiología:

http://astrobionet.rediris.es/

- Psicología de la educación:

http://psicoeduc.rediris.es/

-Farmatoxi. Comunidad virtual de farmacología y toxicología:

http://farmatoxi.rediris.es/

-Comunidad virtual de ciencias del deporte:

http://cdeporte.rediris.es/

-Comunidad virtual de entomología:

http://entomologia.rediris.es/

-Observatorio para la cibersociedad:

http://cibersociedad.rediris.es/

-Área de máquinas y motores térmicos:

http://ammt.rediris.es/

-Proyecto Clío:

http://clio.rediris.es/

-Cálculo simbólico:

http://csimbolico.rediris.es/ 


\subsection{Propuesta de clasificación}

Para efectuar el análisis de los servicios ofertados se ha confeccionado una clasificación ad hoc, para la cual se prestó atención a las propuestas (en concreto, a dos) realizadas previamente por otros autores.

Por una parte, se tuvo presente el trabajo de Fernández, Fernández y Maldonado (2000), que analizan una selección representativa de CVUs profesionales. De partida, estas autoras plantean un esquema general que no se ajusta al que aquí se había establecido. En el caso de ellas, trazan cinco apartados principales: "Datos generales" (de descripción de las comunidades), "Aspectos técnicos de acceso a la información", "Documentación referencial", "Documentación de contenido" y "Servicios", de los que sólo desarrollan los cuatro últimos. Además, priman el mayor número posible de subcategorías (el total final de productos y servicios identificados por separado es de 35), que podrían encuadrarse en tres bloques: uno primero dedicado a las características técnicas de la CVU en el acceso a la información, otro en el que se englobaría de manera general la documentación (tanto primaria como secundaria), y un último que comprendería los servicios ofertados a los usuarios.

Por otra parte, se consideró el estudio de Pazos, Pérez y Salinas (2001) habida cuenta que se centra en las CVUs de RedIris. En este caso hacen una distinción atendiendo a la dependencia que tenga la comunidad virtual respecto de la lista de distribución. A partir de ahí, identifican dos tipos de servicios: los de $\boldsymbol{d o -}$ cumentación, que incluyen enlaces a recursos de interés, revistas electrónicas, archivos de mensajes, o do- cumentos a texto completo; y los de comunicación e intercambio, es decir, lista de distribución, tablón de anuncios, chat, o zona de trabajo colaborativo.

Bebiendo de estos antecedentes, se juzgó conveniente plantear una nueva estructura que estableciera diferentes niveles jerárquicos que permitieran visualizar mejor las herramientas de las CVUs.

\section{«Las CVUs y sus aplicaciones se centran en dos ámbitos: la comunicación y la información; en torno a éstas se ofrecen di- ferentes servicios a los usua- rios con el fin de permitir el tra- bajo colaborativo»}

De esta manera, la clasificación propuesta parte de la idea de que la función principal de las comunidades virtuales de usuarios es la de acoger en un espacio virtual a personas con intereses comunes procurándoles diversas herramientas y servicios para que desarrollen su área de interés. En concreto, las CVUs y sus aplicaciones se centran en dos ámbitos: la comunicación y la información; en torno a éstas se ofrecen las diferentes herramientas y servicios a los usuarios con el fin de permitir el trabajo colaborativo. Sobre esta premisa se ha construido una clasificación que, en un primer nivel, tiene dos categorías: herramientas y servicios. A partir de éstas la clasificación queda como sigue:

\section{Herramientas}

1.1. De información:

-Interna

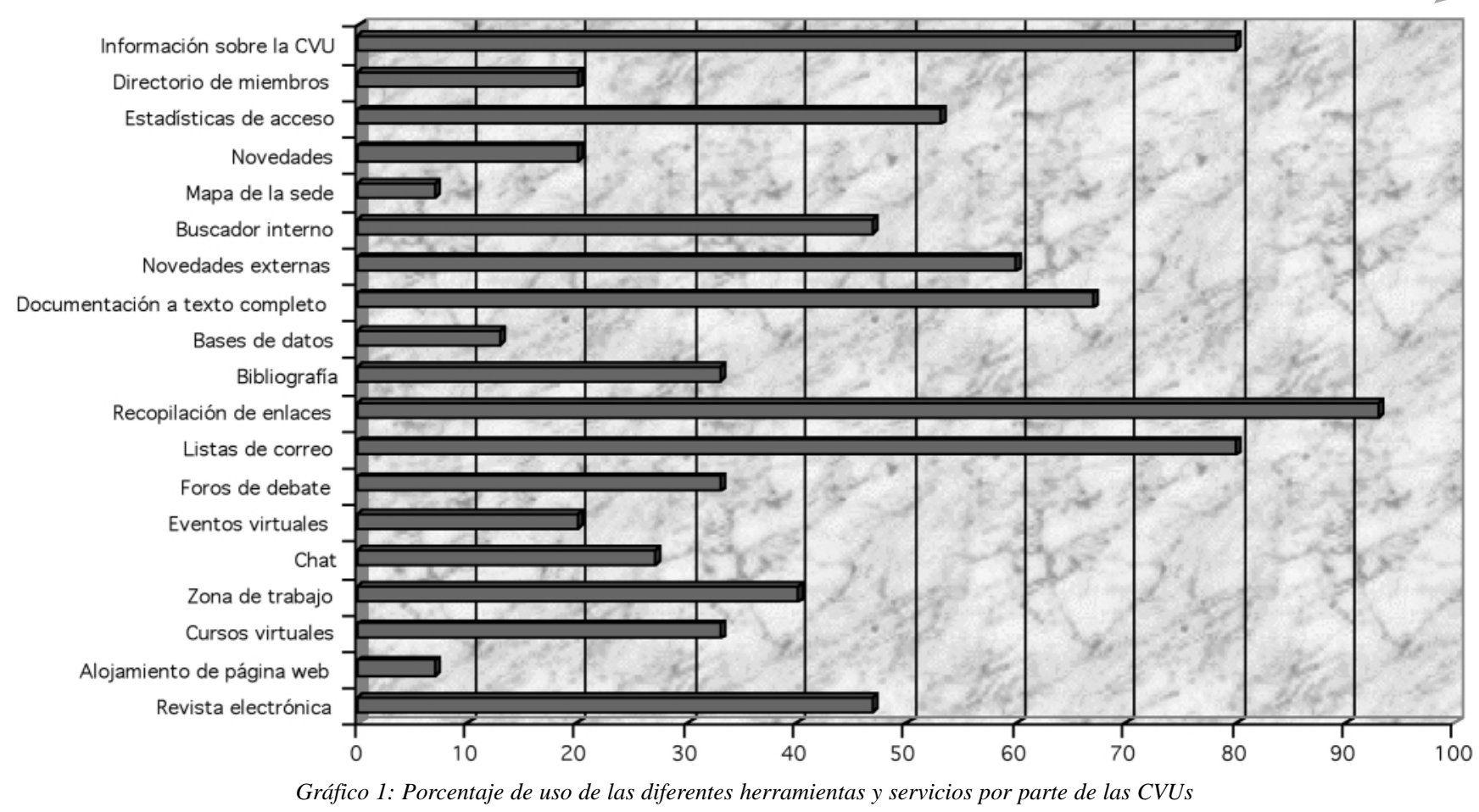




\section{-Externa}

\subsection{De comunicación}

\section{Servicios}

En el caso de las herramientas de información interna se incluyen todas aquellas que hacen referencia a la propia comunidad: funcionamiento, objetivos, novedades, etc. En lo referente a las externas se consideran las que ofrezcan información sobre la temática que congrega a los miembros de la CVU, no ya sobre la propia comunidad.

En cuanto al apartado "Servicios", no se ha incluido dentro de "Herramientas de información" pese a hacer referencia a servicios de información, que es lo que son en esencia. Se ha pensado que, puesto que utilizan tanto herramientas de información como de comunicación para llevarse a cabo, deberían ser considerados como categoría aparte y no incluirse dentro de ninguna de las otras dos.

En lo que se refiere a la nomenclatura utilizada, se denomina categorías a la división principal (herramientas de información, herramientas de comunicación y servicios), subcategorías a la distinción entre herramientas de información interna y externa, y apartados al tercer nivel de división que hace referencia a una herramienta o servicio concreto (listas de correo, cursos virtuales, novedades, etc.). Si el cuadro llegara a desenvolverse en un nivel inferior (por ejemplo, distinguir en la recopilación de enlaces aquellas que describen los recursos de las que no), se designará como subapartados.

En conclusión, en la presente propuesta de cuadro de clasificación de herramientas de servicios de CVUs se quiere identificar aquellos que tengan valor y relevancia por sí mismos, sin caer en la redundancia de una nomenclatura ambigua y confusa, e intentando que sean lo más esclarecedor posible a la hora de dibujar un retrato ajustado de la realidad de cada comunidad.

Así, la estructura propuesta para la clasificación del contenido de las CVUs queda de la siguiente manera:

\section{Herramientas}

\subsection{Herramientas de información}

Bajo este epígrafe se engloban todos aquellos recursos que facilitan información sobre la propia comunidad o que ofrecen documentos (a texto completo o no) relacionados con la temática de la CVU.

\subsubsection{Interna:}

1.1.1.1. Información sobre la CVU: los famosos about omnipresentes en páginas anglosajonas y en los que se informa del origen de la comunidad, de quién hay detrás de ella, de sus objetivos y fines, del perfil de sus miembros, de los servicios ofertados, etc. También se incluyen FAQs (frequent asked questions), preguntas frecuentes con sus correspondientes respuestas referentes a la CVU y a temas prácticos como la manera de hacerse miembro, cómo localizar una información, cómo darse de alta de un servicio, etc. Dentro de este último enfoque habría que considerar guías y tutoriales, que se incluyen más adelante pero que también tendrán cabida en este apartado si su objeto es formar a los miembros en el uso de la comunidad o dirigirlos en su navegación a lo largo de ésta.

\section{«Sorprende el uso marginal (sólo en dos casos, un 13\%) de las bases de datos, propias 0 ajenas, que están accesibles desde la CVU»}

1.1.1.2. Directorio de los miembros: listado alfabético de los miembros de la CVU en la que se aportan datos personales/profesionales y de contacto.

1.1.1.3. Estadísticas de acceso: datos facilitados por los servidores de RedIris en los que se muestra las cifras del acceso a cada CVU en los últimos 12 meses. En teoría estarían muy relacionadas con el epígrafe relativo a la información sobre la propia comunidad, pero en este caso se la ha considerado aparte por tener valor y entidad propia. De cualquier modo, se pueden conocer las estadísticas de acceso de cualquier CVU alojada en RedIris añadiendo "/stats/" al directorio raíz de la URL de cualquiera de ellas.

1.1.1.4. Novedades: noticias sobre novedades, cambios y actualizaciones en la comunidad. Bajo este epígrafe también se encuentran los "tablones de anuncios", pues informan sobre noticias actuales, aunque en los casos que estén centradas en actividades de la CVU. Por último, también se considera el "boletín" de la comunidad, en tanto que publicación de ésta en la que se incluyen novedades referentes a la CVU o a sus áreas de interés.

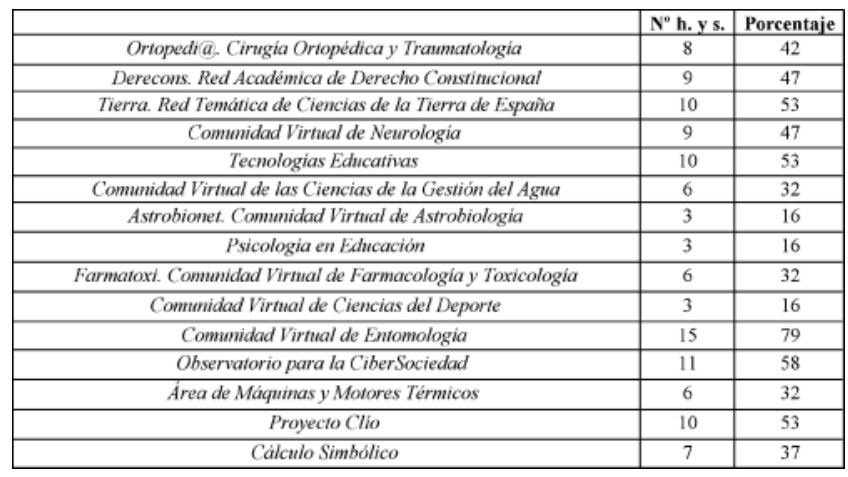

Tabla 3: Número de herramientas y servicios incluidos en cada CVU y porcentaje respecto del total (20) 
1.1.1.5. Mapa de la sede: sistema de ayuda a la navegación que se concreta en la representación gráfica de la estructura de la sede web de la comunidad. Cumple las mismas funciones que un sumario o una tabla de contenido: ofre-

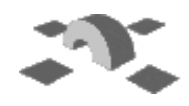
cer una visión global de la web y ayudar en la orientación. Además, el mapa de navegación muestra gráficamente los principales enlaces hipertextuales, seleccionándolos y representando los que se consideran esenciales.

1.1.1.6. Buscador interno: se consideran bajo esta categoría los motores de búsqueda que incluya la CVU y que permiten buscar tanto en las páginas web que configuran la comunidad como en el contenido de los ficheros. No se consideran los buscadores externos al no tener sentido como herramienta de la CVU salvo que ésta pretenda (como lo haría un portal genérico) ofrecer a sus miembros todo lo necesario para trabajar sin que éstos deban salir de ella.

\subsubsection{Externa:}

1.1.2.1. Novedades externas: a pesar del posible solapamiento con el apartado del mismo nombre de la categoría anterior, se incluyen todas aquellas noticias que hagan referencia a novedades profesionales, bibliográficas, o sobre convocatorias laborales o de eventos, o cualquier otro tipo de información de actualidad siempre que el objeto de ésta sea externo a la propia CVU. Sería el caso típico de los "tablones de anuncios", usualmente dedicados a informar sobre novedades externas.

1.1.2.2. Documentación a texto completo: en este caso pueden considerarse dos tipos de documentos a texto completo: de un lado, recopilaciones de textos legales, normativos, reglas, informes, etc.; de otro, repo-

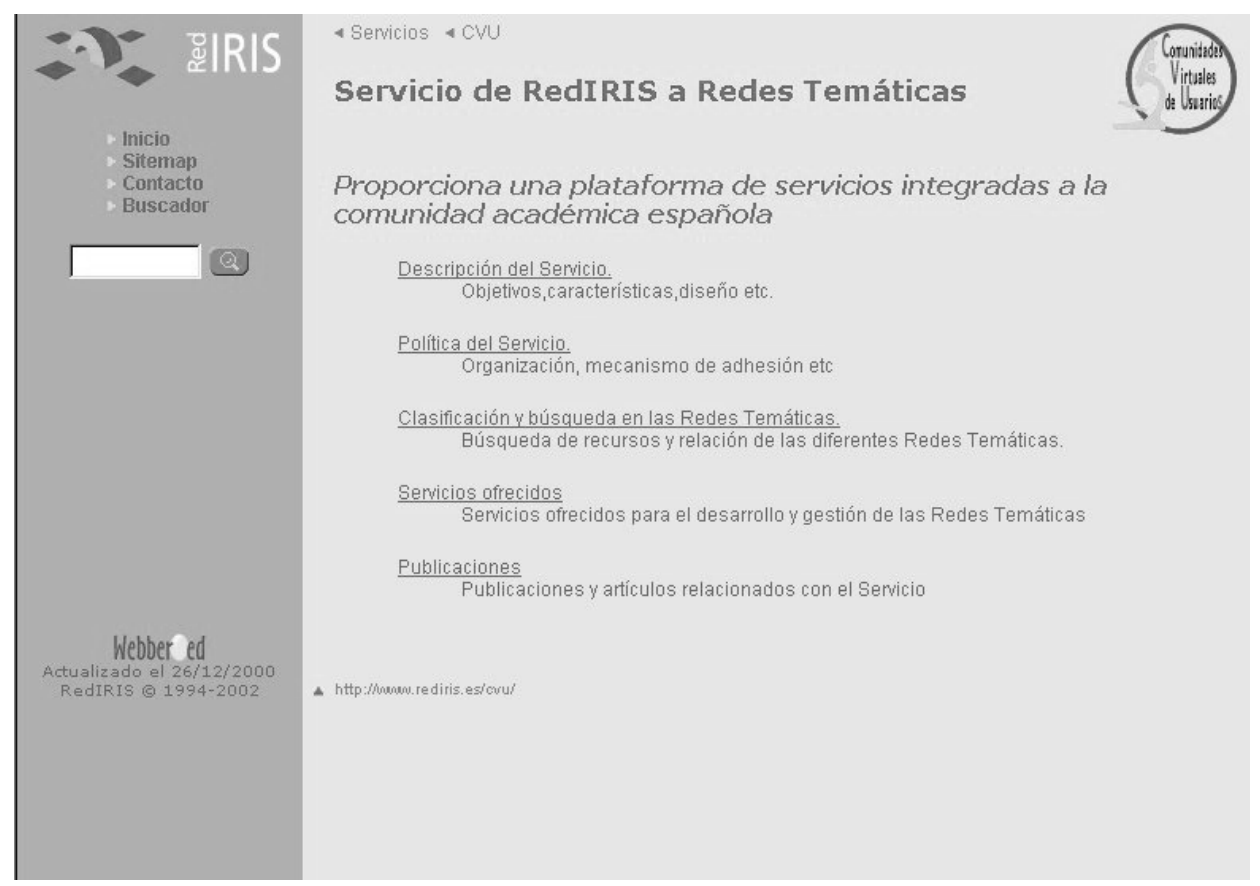

sitorios donde se almacenan a texto completo los trabajos desarrollados por los miembros de la CVU en el marco temático que los ha unido (artículos, estudios, ponencias, ensayos, experiencias, pósteres, etc.). Finalmente, se incluyen en este apartado casos prácticos y experiencias desarrolladas, como, por ejemplo, "Casos clínicos" en la CVU Ortopedi@ ${ }^{8}$.

1.1.2.3. Bases de datos: acceso a bases de datos, básicamente referenciales, producidas por miembros de la comunidad o por otros organismos.

1.1.2.4. Bibliografía: relación alfabética de publicaciones seriadas o monografías relativas al área de interés de la CVU; en algún caso puede presentarse bajo la forma de catálogo o dando acceso a catálogos de bibliotecas o librerías (Amazon, principalmente).

1.1.2.5. Recopilación de enlaces: catálogo o gestor de recursos digitales, directorio o listado, donde se recogen enlaces a entidades, servicios, información, referencia sobre el área de interés de la CVU; esto incluye las revistas electrónicas, accesibles a texto completo. Los enlaces pueden estar descritos o comentados, y pueden hacer referencia a la temática de la comunidad o ser referenciales (diccionarios, atlas, guías de internet, etc.).

\subsection{Herramientas de comunicación}

Las que permiten comunicarse a los miembros sincrónica o asincrónicamente, y de uno a uno o de uno a muchos.

1.2.1. Listas de correo: distribuyen a todos los miembros los mensajes de cualquier tipo enviados a una dirección electrónica del servidor. Su función es hacer público cualquier propuesta, necesidad, o comentario. Aunque pueden desarrollarse debates, no se incluye en este apartado los denominados "foros de debate".

1.2.2. Foros de debate: suelen ser un área restringida en la que los miembros, o grupos de trabajo, a través de una lista de distribución, discuten a propósito de un tema de la comunidad virtual. Dentro de cada discusión pueden seguirse las diferentes líneas debatidas gracias a una estructura jerárquica formada por los encabezamientos de los mensajes.

1.2.3. Eventos virtuales: congresos, simposios, jornadas, 
mesas redondas de la temática de la CVU y que se llevan a cabo en línea.

1.2.4. Chat: nombre genérico de los sistemas multiusuario de discusión en línea a través de internet que permiten la comunicación (escrita) sincrónica entre dos o más personas.

1.2.5. Zona de trabajo: espacio compartido por los miembros que permite la realización de actividades de forma colaborativa a través de la comunidad virtual. Para el caso que nos ocupa, se referiría a las aplicaciones de $B s c w^{9}$.

\section{Servicios}

Servicios de información interactivos que ofrece la comunidad a sus miembros y que funcionan por medio de la filosofía push.

2.1. Cursos virtuales: bajo este epígrafe se incluyen todas las actividades formativas desarrolladas en línea y para los miembros de la CVU; pueden ser interactivos o simplemente ficheros planos; pueden ser referentes a temas relacionados con el ámbito de la comunidad o sobre contenidos más generales (cómo buscar información en internet, o cómo elaborar una página web, por ejemplo).

2.2. Alojamiento de páginas web: posibilidad de los usuarios de crear una página web en la CVU, si bien por lo general suelen ser desarrolladas por grupos de investigación en forma de portal temático dentro de la comunidad para informar y dar acceso a novedades, documentos y proyectos que llevar a cabo relacionado todo ello con su ámbito de trabajo. Pueden considerarse el "escaparate público" de las zonas de trabajo (restringidas) de los diferentes grupos; en algunos casos aparecen bajo la denominación de "observatorios".

2.3. Revista electrónica: publicación seriada elaborada por los miembros de la CVU, que puede tener material propio o de autores ajenos a la comunidad y que, a diferencia de los repositorios de documentos a texto completo (caso de la Tribuna del ortonauta) ${ }^{10}$, deben seguir una numeración correlativa y además suelen tener asignado un Issn.

Partiendo, pues, de esta estructura, se ha procedido al vaciado de las herramientas y servicios de las CVUs seleccionadas, asignándolos unívocamente a una categoría, apartado o subapartado.
Senvicios 4 CVU 4 Buscar

Servicio de indexación en las Redes Temáticas de RedIRIS

Búsqueda en las Redes Temáticas de RedIRIS

Ayuda · Relación actual de CVUs

Ciencias agrarias

- Antropología

- Astronomía, astrofísica

Ciencias de la tierra v del

Ciencias de las artes y las letras

- Ciencias de la vida

Ciencias económic

Ciencias tecnológicas

- Demografía

Ciencias iurídicas y

derecho

Ciencias de la Educación

Documentación

- Estadística

- Ética

- Lógica

Matemáticas

Física

- Geografía - Historia - Linqüística - Ciencias Políticas - Psicología - Química Ciencias Sociales Ciencias del Deporte Música

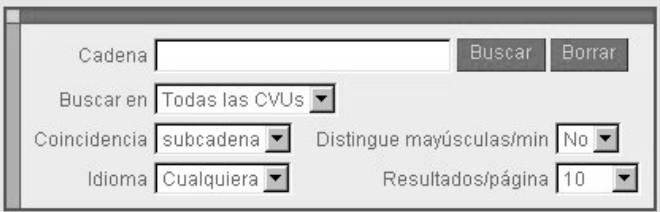

Este Buscador ha sido construido usando el software Hawest uede acceder a información sobre cómo formular prequntas

Por otra parte, después de vaciar las CVUs objeto de análisis se ha observado que cuatro de los apartados considerados a priori debían rechazarse por no aparecer en ninguno de los casos propuestos. En concreto, el primero hace referencia a una herramienta de comunicación, y los otros a servicios de información:

Correo electrónico: mediante un programa específico, permite disponer de un buzón para remitir y recibir mensajes entre usuarios de la red (en este caso, de la comunidad virtual). No debe ofrecerse en las CVUs de RedIris al considerar la necesidad de que el usuario disponga ya de una cuenta de correo propia para poder acceder y utilizar las herramientas y servicios de la comunidad.

Área de descarga de software: servicio que permite la descarga de software con el fin de facilitar el trabajo colaborativo o por resultar de interés profesional.

\section{«La comunicación en las comu- nidades alojadas en Redlris es- tá centrada en las listas de co- rreo, a juzgar por el porcentaje $(80 \%)$ con el que aparecen en las CVUs»}

Alerta: servicio que consiste en el envío de novedades por correo electrónico referentes tanto a la propia CVU como externas (por ejemplo, cambios experimentados en la comunidad según suceden, o relación actualizada de convocatorias públicas de empleo conforme se hacen públicas).

Difusión selectiva de la información (DSI): en este caso, el envío de novedades por correo electróni- 
co se concreta a partir de unos perfiles definidos por los miembros de la CVU.

El análisis y tratamiento de los datos ha dado lugar a dos tipos de cuadros: uno que cuantifica el uso de cada herramienta y servicio por parte de las CVUs, y otro que muestra la envergadura de cada comunidad virtual en función de cuántas herramientas y servicios incluyen (no se entra en la manera como los utilizan).

Por último, en el presente estudio no se ha aplicado ningún sistema de control de fiabilidad de la observación que se ha llevado a cabo: test-retest, test-test (el más pertinente para aplicar en este trabajo), y test-norma. De ahí que sufra las limitaciones propias de esta ausencia y los resultados puedan estar sesgados.

\section{Resultados y discusión}

En la tabla 1 se muestra la presencia o ausencia de cada una de las 19 herramientas y servicios definidos en las 15 CVUs de RedIris seleccionadas.

En las tablas 2 y 3 pueden verse, respectivamente:

- las frecuencias y porcentajes de uso de cada una de ellos (así como de las categorías y subcategorías) por parte de las CVUs. mismos.

- las frecuencias (y los porcentajes) de uso de los

La media ofertada es de 8 herramientas o servicios por cada CVU, lo que equivale al $42 \%$.

\subsection{Herramientas de información}

Esta categoría es la que comprende las más utilizadas en las comunidades; tanto es así que la totalidad de las CVUs incluyen alguna herramienta de este tipo. Ello se plasma tanto en la extensa tipología definida (hasta 11 diferentes) frente a las otras categorías, como en la presencia destacada de herramientas de información entre las más utilizadas (la recopilación de enlaces, sobre todo, y la información sobre la CVU).

\subsubsection{Interna}

Siguiendo con la línea anterior, una de ellas es la información sobre la propia comunidad, que aparece hasta en un $80 \%$ de CVUs. Ello puede deberse, además de otras razones obvias, a la gran variedad de casuísticas que contempla ese apartado: los about, las $F A Q$ s, guías, manuales y tutoriales, etc.

Le siguen las estadísticas de acceso $(53 \%$ de las comunidades). En este caso se han considerado aquellas que mostraran esta opción de manera explícita, puesto que, como ya se comentó, todas tienen disponibles sus estadísticas añadiendo "/stats/" al directorio raíz de las URLs. No se han incluido las que sólo ofrecían estadísticas de algún servicio concreto de la CVU, como, por ejemplo, la de entomología, que incluye una opción dentro del chat para consultar las que hacen referencia a éste.

Finalmente, merece destacarse el buscador interno (un $47 \%$ de los casos), que, salvo casos aislados (Farmatoxi utiliza Google como motor de búsqueda interno) ${ }^{11}$, remiten al que dispone RedIris para buscar dentro de todas las comunidades que aloja y que utiliza el software Harvest ${ }^{12}$. Se trata éste de un grupo integrado y gratuito de herramientas para recopilar, extraer, organizar, buscar, guardar en la memoria de su sistema, y replicar información relevante localizada a lo largo de internet. Fácil de utilizar, permite procesar información en muy diferentes formatos y desde ordenadores muy distintos. Como plataforma, trabaja en Windows $N T$ y en Unix. Ha sido implantado por la Universidad de Hannover en su página web ${ }^{13}$ y por el Index morganagus $^{14}$.

En otro orden, el mapa de la web sólo es utilizado en una ocasión (Entomología) $)^{15}$; su ausencia en las otras CVUs puede deberse a cierta dejadez en el diseño de la usabilidad.

\subsubsection{Externa}

Las herramientas que ofrecen información externa se incluyen en el $100 \%$ de las comunidades, frente al $87 \%$ de la subcategoría anterior. La explicación es contundentemente sencilla: un $93 \%$ de las CVUs ofrecen una recopilación de enlaces (descritos o no) a recursos de interés para la temática en la que se centra.

En segundo lugar se encuentran los archivos de documentación a texto completo (un 67\%) propia de los miembros de la comunidad o de autoría ajena a ésta pero no al tema que estudia. Con una frecuencia similar (el 60\% de las CVUs) se encuentran las novedades externas, las noticias sobre eventos, cursos, simposios, convocatorias, actividades de todo tipo relacionadas con el ámbito de la comunidad pero desarrolladas fuera de ésta.

\section{«Si bien las aplicaciones de in- formación externa son las más desarrolladas, los apartados predominantes son (en orden descendente): recopilación de enlaces, información sobre la CVU / listas de correo, docu- mentación a texto completo»}

Sorprende el uso marginal (sólo en dos casos, un $13 \%$ ) de las bases de datos, propias o ajenas, que están accesibles desde la CVU. Seguramente es un indicio 
del poco tratamiento que recibe la información en las redes temáticas analizadas.

\subsection{Herramientas de comunicación}

Son aprovechadas, como media, por un $93 \%$ de las CVUs, que no se aleja mucho de las categorías anteriores, pero no demuestra ser el propósito principal por el que son utilizadas las CVUs.

La comunicación en las comunidades alojadas en RedIris está centrada en las listas de correo, a juzgar por el porcentaje $(80 \%)$ con el que aparecen en las CVUs. Claro que esta cifra es ciertamente engañosa, puesto que, salvo en tres casos (Astrobiología, Observatorio para la cibersociedad y Área de máquinas y motores térmicos), es el origen de la CVU, tal y como se ha explicado en el apartado correspondiente al marco del objeto de estudio.

\section{«Considerando la política de creación y desarrollo de CVUs planteada por Redlris, se ob- serva una dependencia excesi- va de éstas hacia las listas de correo de las que proceden»}

Asombra, sin embargo, que la zona de trabajo sólo se encuentre en un $40 \%$ de los casos, considerando que ha de ser la herramienta más completa (e imprescindible) para desarrollar el trabajo conjunto en línea. De esta manera se limitan mucho las posibilidades de las comunidades, quedando como páginas planas y unidireccionales.

En el extremo inferior aparecen los eventos virtuales, sólo presentes en un $20 \%$ de las CVUs a pesar de ser uno de los objetivos que se propone RedIris con el desarrollo de las Redes temáticas ${ }^{16}$.

\subsection{Servicios}

Superando la cifra alcanzada en el caso examinado por Fernández, Fernández y Maldonado (2000), los servicios aparecen en dos tercios de las CVUs aquí estudiadas $(67 \%)$.

Entre los diferentes apartados destaca el desarrollo de revistas electrónicas (47\%), que muchas veces hacen las funciones de repositorio de documentación a texto completo y de novedades internas y externas, fundamentalmente. El alojamiento de página web para sus miembros o grupos de trabajo internos se da en contadas ocasiones ( 4 casos, un $27 \%$ del total) y no pocas veces para cumplir lo mismo que se ha planteado en las revistas electrónicas.

Por último, a título anecdótico, mencionar la casi nula presencia de cursos virtuales (sólo en un caso, en
Entomología $)^{17}$, puesto que la mayoría de guías, manuales y tutoriales hacen referencia a la navegación de la propia CVU y al uso de herramientas de que dispone.

En cuanto a las comunidades virtuales estudiadas, se observa (tal y como muestra la tabla 3) que sólo 6 (poco más de un tercio) usan más de la mitad de las herramientas y servicios disponibles, pero que ninguna alcanza el 60\%, salvo una, Entomología, que destaca de manera notable y aplica en su área no restringida casi el $80 \%$ de los diferentes tipos de herramientas y servicios utilizados en el conjunto de las CVUs de RedIris.

«Se desaprovechan e infrautili-
zan las posibilidades de traba-
jo, de investigación colaborati-
va y de creación de contenidos
cooperativamente que brindan
las infraestructuras que Redlris
ofrece a la comunidad acadé-
mico-científica española para el
desarrollo de comunidades vir-
tuales»

En definitiva, en lo referente al cuadro de clasificación que se ha propuesto en el presente artículo, se reconoce cierta problemática al englobar la oferta de herramientas y servicios en 15 categorías (frente a las 35 que proponen Fernández, Fernández y Maldonado). De esta manera, se han creado parámetros que quizás podrían tildarse de excesivamente generales, no permitiendo un análisis a pequeña escala y así poder evaluar con mayor precisión la calidad de las diferentes CVUs. Ejemplo de ello sería incluir subcategorías para distinguir si las recopilaciones de recursos incluyen descripciones o no, en este caso concreto.

\section{Conclusiones}

La realidad actual de las CVUs académico-científicas españolas alojadas en RedIris arroja la siguiente imagen: si bien las herramientas de información externa son las más desarrolladas, los apartados predominantes son (en orden descendente):

—Recopilación de enlaces

—Información sobre la CVU / Listas de correo

-Documentación a texto completo

La conclusión parece bastante clara: considerando la política de creación y desarrollo de CVUs planteada por RedIris, se observa una dependencia excesiva de éstas hacia las listas de correo de las que proceden (condición sine qua non para la creación de CVUs). Así, la mayoría, al dar el salto desde una lista que al 
parecer se quedaba pequeña para los intereses de sus usuarios, sólo ha llegado a alcanzar una página web que, además de hablar de sí misma y de sus intenciones, recoge algunos recursos en línea y textos desarrollados por los miembros de esa comunidad virtual. Además, claro está, de un enlace explícito a la lista de distribución de la que proviene.

Observado estrictamente, no podría considerarse como comunidad virtual a casi la mitad de las que han sido analizadas en el presente estudio: habría que dar la razón a Fernández, Fernández y Maldonado (2000) y considerarlas como portales temáticos sin mayor aspiración.

Se coincide, asimismo, con estas autoras cuando destacan en sus conclusiones que, por un lado, ninguna de las comunidades analizadas llega a desplegar todos los productos y servicios que debería incluir una CVU ideal, y, por otro, se constata el subdesarrollo e infrautilización de los servicios como alertas, DSIs, asesorías y similares frente a los productos documentales (referenciales o a texto completo) o informativos tradicionales.

Por otra parte, y siguiendo los estudios y propuestas de Hawa-Attourah (1999: 448) y de Cothrel (1999a), se ha observado en las CVUs una dispersión y redundancia de herramientas y servicios dentro de las mismas comunidades virtuales pero con diferentes denominaciones, lo que puede ser confuso de cara al usuario. Por ello, hay que tener presente de buen principio qué es lo que se quiere obtener con la CVU (Lewis, 2001) y, a partir de ahí, sondear qué herramientas y servicios satisfarán con mayor eficacia las necesidades de los usuarios sin redundancias ni confusiones.

\section{«La gran mayoría de CVUs de- bería replantearse no ya su es- tructura y contenidos, sino si realmente sabe lo que busca al- canzar por medio de la comuni- dad virtual, lo que precisan sus usuarios y cómo gestionar las CVUs para satisfacer tales ne- cesidades»}

Del mismo parecer son Pazos, Pérez y Salinas (2001), que insisten en que la clave para que funcione la comunidad reside en la participación, en la colaboración; pero, sobre todo, para lograr unos niveles eficientes de interacción debe hacerse uso de la CVU con un fin o interés concreto que ayude a generar un sentido de pertenencia a ésta y, con ello, una mayor implicación.
Finalmente, los servicios de información, paradigmáticos del desarrollo tecnológico alcanzado por la biblioteconomía y la documentación, apenas aparecen ofertados por ser los menos automatizados y requerir una mayor implicación del staff de cada CVU ya que se trata de tareas que precisan de una inversión altruista de tiempo y esfuerzo personal.

Se observa, por tanto, que se desaprovechan e infrautilizan las posibilidades de trabajo, de investigación colaborativa y de creación de contenidos cooperativamente que brindan las infraestructuras que RedIris ofrece a la comunidad académico-científica española para el desarrollo de comunidades virtuales. Aunque este panorama no es exclusivo de las CVUs de $R e$ dIris, como se ha visto que mostraban otros estudios (Fernández, Fernández y Maldonado (2000)).

Para terminar, al observar los pobres resultados obtenidos por la mayoría de CVUs de RedIris, habría que plantearse si la red académica española no debería tomar medidas más expeditivas en los casos que ella misma supone: "La dirección de RedIris tiene competencia para plantearse y estudiar la posibilidad de denegar el servicio si:

- En el transcurso de un período de tiempo suficiente se detecta que los objetivos iniciales de adhesión no se han cumplido.

- Los servicios ofrecidos son utilizados con otros fines que los pactados en el contrato de adhesión.

- La información contenida en los diferentes servicios (páginas web, directorio, etc.) no está completamente actualizada.

- Se detectan graves abusos en la dirección de la CVU, infringiendo el objetivo primordial del servicio: dirección cooperativa y trabajo en grupo"18.

Lejos de obtener los objetivos primigenios planteados por RedIris y para cada caso concreto, la gran mayoría de CVUs debería replantearse no ya su estructura y contenidos, que es evidente, sino si realmente sabe lo que busca alcanzar por medio de la comunidad virtual, lo que precisan sus usuarios y cómo gestionar las CVUs para satisfacer tales necesidades.

\section{Notas}

\footnotetext{
1. Para el caso español pueden considerarse: Castillo (1999a y 1999b); Fernández, Fernández y Maldonado (2000); Riera (2000); Medrano y Suárez (2001); Pazos, Pérez y Salinas (2001); Sánchez y Saorín (2001); Sanz de las Heras (2001); Cobos y Alamán (2002); Ontalba (2002b)

2. http://www.rediris.es/cvu/

3. Un caso paradigmático es el de IweTel (Ontalba, 2002a).

4. http://www.rediris.es/cvu/descri/

5. Puede consultarse el listado casi completo en:
} 
http://www.rediris.es/cvu/rel.es.html.

Aun así, no aparecen todas las CVUs, pues algunas se encuentran en el Área temática correspondiente.

6. http://www.rediris.es/cvu/buscar/

7. http://www.rediris.es/cvu/rel.es.html

8. http://ortopedia.rediris.es/casos/casos.html

9. http://www.rediris.es/cvu/serv/bscw/

10. http://ortopedia.rediris.es/tribuna/tribuna.html

11. http://farmatoxi.rediris.es/buscar.htm

12. http://www.rediris.es/CVU/buscar/

13. http://harvest.rrzn.uni-hannover.de/

14. http://sunsite.berkeley.edu/\%7Eemorgan/morganagus/index.html

15. http://entomologia.rediris.es/mapas.htm

16. http://www.rediris.es/CVU/descri/

17. http://entomologia.rediris.es/introento/index.htm

18. http://www.rediris.es/cvu/descri/org.es.html

\section{Bibliografía}

Bond, Oliver (1998). "Virtual communities". En: Managing information, 1998 , v. 5 , n. 10 , pp. 39-41.

Burnett, Gary (2000). "Information exchange in virtual communities: a typology". En: Information research, 2000, July, v. 5, n. 4. Consultado en: 23-04-01.

http://informationr.net/ir/5-4/paper82.html

Castillo Vidal, Jesús (1999a). "Trabajo colaborativo en comunidades virtuales". En: El profesional de la información, 1999, noviembre, v. 8, n. 11, pp. $40-47$.

Castillo Vidal, Jesús (1999b). “Aplicación de herramientas groupware a través de internet: Bscw. Su utilización en las comunidades virtuales de usuarios". En: Boletín de la RedIris, 1999, n. 50-51, pp. 23-25.

Cobos, Ruth; Alamán, Xavier (2002). "Cristalización del conocimiento en una comunidad de usuarios”. En: Interacción 2002: III Congreso internacional de interacción persona-ordenador, 2000. Leganés (Madrid): Asociación Interacción Persona-Ordenador (Aipo), pp. 128-135.

Cothrel, Joseph; Williams, Ruth L. (1999a). "On-line communities: helping them form and grow". En: Journal of knowledge management, 1999, v. 3 , n. 1 , pp. 54-60.

Cothrel, Joseph (2000a). "Measuring the success of an online community". En: Strategy and leadership, 2000, v. 28, n. 3. Consultado en: 0504-02.

http://www.participate.com/research/art-measuresuccess.asp

Cothrel, Joseph (2000b). "Creating functional online communities". En: Digitrends, 2000, December 14. Consultado en: 05-04-02. http://www.digitrends.net/ebiz/13643_12792.html

Fernández Sánchez, Elena; Fernández Morales, Isabel; Maldonado Martínez, Ángeles (2000). "Comunidades virtuales especializadas: un análisis comparativo de la información y servicios que ofrecen al usuario". En: VII Jornadas españolas de documentación: la gestión del conocimiento: retos y soluciones de los profesionales de la información, 2000. Bilbao: Aldee, pp. 383-390.

Gardner, Tracy; Russell, Rosemary (1999). “A collaborative workspace environment: experience of evaluation and selection in the Agora project". En: Vine, 1999, n. 109, pp. 16-26.

Gisbert, M.; Feliu, Víctor (2000). “Para qué sirven las listas de distribución?”. En: III Congreso internacional de comunicación, tecnología y educación: redes, multimedia y diseños virtuales, 2000. Oviedo: Universidad de Oviedo.

Hawa-Attourah, Marc (1999). "La Red a la carta: las comunidades virtuales de usuarios y los servicios telemáticos temáticos integrados". En:
Primer congreso virtual iberoamericano de neurología; y en: Revista de neurología, 1999, n. 29, pp. 486-490. Consultado en: 17-01-02.

http://www.revneurol.org/web/2905/h050486.pdf

http://www.uninet.edu/neurocon/congreso-1/conferencias/int-neurohawa.html

Iparraguirre, Jordi (1998). Workshop on virtual communities, 1998, mayo. Consultado en: 24-05-01.

http://www.gpd.org/maig98/eng/comvirture.htm

[Consultado a través de http://www.archive.org]

Jiménez, J. A.; Padilla, A. (1998). "Análisis estratégico de la integración de las listas de distribución en las comunidades virtuales de usuarios". En: Boletín de la RedIris, 1998, n. 46-47, pp. 107-114.

Levy, Philippa (1999). "Virtual Communities and information services: an overview". En: Vine, 1999, n. 109, pp. 3-9.

Lewis, Robert (2002). Grupos de trabajo en comunidades virtuales. Consultado en: 22-04-01.

http://www.uoc.es/web/esp/art/uoc/lewis0102/lewis0102_imp.html

Medrano Corrales, Isabel; Suárez Samaniego, Luis (2001). Ensayo sobre estrategias para documentar el conocimiento en una organización, 2001. Consultado en: 12-12-01.

http://www.gestiondelconocimiento.com/documentos2/imedrano/document ar.htm

Ontalba y Ruipérez, José Antonio (2002a). "Análisis de contenido de la lista de distribución IweTel (1998-2000)". En: El profesional de la información, 2002, enero-febrero, v. 11, n. 1, pp. 14-27. Consultado en: 12-03-02. http://www.rediris.es/CVU/publ/EPI.pdf

Ontalba y Ruipérez, José Antonio (2002b). Las comunidades virtuales como herramientas para la socialización del conocimiento tácito. Máster documentación digital, Universitat Pompeu Fabra, 2002, febrero. Consultado en: 29-04-02. http://www.editaweb.com/docdigital/

Ordinas, Catalina; Pérez Garcias, Adolfina; Salinas, Jesús (1999). “Comunidad virtual de tecnología educativa. Edutec". En: Edutec'99. IV Congreso internacional de tecnología, educación y desarrollo sostenible, 1999. Sevilla: Universidad de Sevilla. Consultado en: 03-08-02 http://www.uib.es/depart/gte/edutec99/comunidad.html

Pazos, María; Pérez Garcias, Adolfina; Salinas, Jesús (2001). “Comunidades virtuales: de las listas de discusión a las comunidades de aprendizaje". En: Edutec'01. V Congreso internacional de tecnología, educación y desarrollo sostenible, 2001. Murcia. Consultado en: 03-08-02. http://gte.uib.es/articulo/CVIRTUALES01.pdf

Pérez Garcias, Adolfina; Salinas, Jesús (2001). “Comunidades virtuales al servicio de los profesionales: Edutec, la comunidad virtual de tecnología educativa". En: Educación y biblioteca, 2001, abril, n. 122, pp. 58-63.

Pimienta, Daniel; Dhaussy, Catherine (2002). "La metodología Emec: gestión del conocimiento en una comunidad virtual latinoamericana". En: Novática: revista de la Asociación de Técnicos de Informática, 2002, enero-febrero, n. 155, pp. 51-55.

Riera Barsallo, Patricia (2000). "Servicios de información a medida para una comunidad virtual”. En: XI Jornadas bibliotecarias de Andalucía, 2000, pp. 275-285.

Sánchez Arce, Ma Vanessa; Saorín Pérez, Tomás (2001). "Las comunidades virtuales y los portales como escenarios de gestión documental y difusión de información”. En: Anales de Documentación, 2001, n. 4, pp. 215-227. Consultado en: 08-06-01.

http://www.um.es/fccd/anales/ad04/a12comvirtuales.pdf

Sanz de las Heras, Jesús (2001). "Plataforma de Servicios de RedIris a las redes temáticas científicas". En: Jornada d'Aplicacions del Bscw en la docència i la recerca a la universitat, 2002, 31 de enero. Consultado en: 23-03-02. http://terra.d5.ub.es/pub/bscw.cgi/d922993/Plataforma\%20Telem\%e1tica\%20de\%20RedIRIS

http://www.rediris.es/CVU/plata/plataforma.htm

José Antonio Ontalba y Ruipérez. Biblioteca Virtual. Universitat Oberta de Catalunya.

jontalba@uoc.edu 